S1 Table. Characteristics of human cases due to Chrysomya bezziana myiasis recorded worldwide.

\begin{tabular}{|c|c|c|c|c|c|c|c|c|c|c|c|}
\hline ID & Age & Sex & Year & Country & Location & Socioeconomic status & Site of infestation & Methods for removal of larvae & Underlying diseases & Clinical signs and symptoms & Outcome \\
\hline $1^{70}$ & 49 & F & 2004 & China & Hong Kong & The elderly home & Torso: left breast & Surgical removal & Left breast cancer & Bleeding, discharge, uler, noticed larvae & $\mathrm{NR}$ \\
\hline $2^{71}$ & 81 & $\mathrm{~F}$ & 2005 & China & Hong Kong & The elderly home & Mouth & NR & Bedridden, ulcer & Bleeding, noticed larvae & Death (septicemia)** \\
\hline $3^{72}$ & 79 & $\mathrm{~F}$ & 2005 & China & Hong Kong & The elderly home & Mouth & $\mathrm{NR}$ & Bedridden & Bleding, chills, noticed larvae & Death $(\mathrm{NR})^{* * *}$ \\
\hline $4^{73}$ & 78 & $\mathrm{~F}$ & 2005 & China & Hong Kong & $\begin{array}{l}\text { An extended care } \\
\text { hospital }\end{array}$ & Mouth & Surgical removal & $\mathrm{NR}$ & Swollen, bleeding & Stable \\
\hline $5^{74}$ & 50 & $\mathrm{~F}$ & 2005 & China & Hong Kong & $\mathrm{NR}$ & Limb: left lower limb & NR & Ulcer & $\mathrm{NR}$ & Stable \\
\hline $6^{75}$ & 66 & M & 2006 & China & Hong Kong & $\mathrm{NR}$ & Scalp & $\mathrm{NR}$ & Scalp wound & Noticed larvae & Stable \\
\hline $7^{76}$ & 49 & M & 2006 & China & Hong Kong & $\mathrm{NR}$ & $\begin{array}{l}\text { Perineal \& inguinal regions: } \\
\text { groin }\end{array}$ & $\mathrm{NR}$ & $\mathrm{NR}$ & Pain, noticed larvae & Stable \\
\hline $8^{77}$ & 79 & м & 2006 & China & Hong Kong & The elderly home & Mouth & Surgical removal & Bedridden & Bleeding, ulcer, noticed larvae & Stable \\
\hline $9^{78}$ & 93 & $\mathrm{~F}$ & 2007 & China & Hong Kong & The elderly home & Mouth: right lower gum & Surgical removal & Bedridden, stroke & Bleeding, fever & Stable \\
\hline $10^{79}$ & 49 & м & 2010 & China & Hong Kong & $\mathrm{NR}$ & Torso: right shoulder & $\mathrm{NR}$ & Nasopharyngeal carcinoma, slipped and fell into a valley, a shoulder wound & Noticed larvae & Stable \\
\hline $11^{80}$ & 31 & $\mathrm{~F}$ & 2010 & China & $\begin{array}{l}\text { Hong Kong } \\
\text { (Indonesian) }\end{array}$ & $\mathrm{NR}$ & Torso: back & NR & Infection: ruptured back mass, wound & Bleeding, discharge, noticed larvae & Stable \\
\hline $12^{80}$ & 93 & $\mathrm{~F}$ & 2010 & China & Hong Kong & The elderly home & Mouth & $\mathrm{NR}$ & Multiple underlying illnesses, bedridden, dementia, tube feeding & Fever, ulcer & $\begin{array}{l}\text { Death (underlying } \\
\text { illlnesses)** }\end{array}$ \\
\hline $13^{29}$ & 78 & $\mathrm{~F}$ & 2010 & China & Hong Kong & The elderly home & Mouth & $\mathrm{NR}$ & Bedridden & Bleeding, noticed larvae & Discharged \\
\hline $14^{29}$ & 81 & м & 2010 & China & Hong Kong & The elderly home & Torso: sacral sore & $\mathrm{NR}$ & Bedridden, dementia, multiple bed sores, infections & Noticed larvae & Death $(\mathrm{NR})^{* *}$ \\
\hline $15^{29}$ & 80 & $\mathrm{~F}$ & 2010 & China & Hong Kong & The elderly home & Tracheostomy & $\mathrm{NR}$ & Bedridden, tracheostomy wound & Bleeding, noticed larvae & Stable \\
\hline $16^{81}$ & 89 & $\mathrm{~F}$ & 2010 & China & Hong Kong & $\mathrm{NR}$ & Limb: left foot & $\mathrm{NR}$ & Slipped and an injury over her leff foot, infection: later developed gangrene, wound & Necrosis & Stable \\
\hline $17^{81}$ & 88 & $\mathrm{~F}$ & 2010 & China & Hong Kong & The elderly home & Mouth & NR & Bedridden, tube feeding & Bleeding, noticed larvae & Stable \\
\hline $18^{25}$ & 89 & $\mathrm{~F}$ & 2011 & China & Hong Kong & Home & Limb: right foot & $\begin{array}{l}\text { Surgical removal, below knee amputation due to extensive } \\
\text { ulceration }\end{array}$ & Multiple underlying illnesses, hypertension, stroke, diabetes mellitus, diabetic ulcer & Noticed larvae, severe tissues and/or bone destruction & Stable \\
\hline $19^{82}$ & 83 & $\mathrm{~F}$ & 2011 & China & Hong Kong & The elderly home & Limb: right foot & Manual removal, surgical removal & Peripheral vascular disease with chronic ulcers on both legs & Noticed larvae & Stable \\
\hline $20^{83}$ & 82 & м & 2011 & China & Hong Kong & home & Limb: right toe & Manual removal & Multiple underlying illnesses, dementia, hypertension, stroke, right toe ulcer & Noticed larvae, fever & Recovery/discharged \\
\hline $21^{84}$ & 80 & $\mathrm{~F}$ & 2011 & China & Hong Kong & $\mathrm{NR}$ & Mouth: gum & Manual removal & Multiple underlying illnesses, bedridden & Bleeding, noticed larvae & Recovery/discharged \\
\hline $22^{24}$ & 82 & м & 2011 & China & Hong Kong & The elderly home & Mouth & Manual removal & $\begin{array}{l}\text { Multiple underlying illnesses, bedridden, stroke, dementia, hypertension, chest } \\
\text { infection }\end{array}$ & Fever, swollen, noticed larvae & Death $($ Pneumonia)** \\
\hline $23^{85}$ & 94 & $\mathrm{~F}$ & 2011 & China & Hong Kong & The elderly home & Mouth & Manual removal & Dementia,bedridden & Fever, noticed larvae & Stable \\
\hline $24^{86}$ & 94 & $\mathrm{~F}$ & 2011 & China & Hong Kong & The elderly home & Ear & Manual removal & $\begin{array}{l}\text { Multiple underlying illnesses, ear infection: chronic otitis media, hypertension, } \\
\text { stroke, dementia, bedridden }\end{array}$ & Discharge, noticed larvae & Stable \\
\hline $25^{5^{87}}$ & 79 & $\mathrm{~F}$ & 2011 & China & Hong Kong & The elderly home & Mouth & Manual removal & Multiple underlying illnesses, tube feeding, hypertension, stroke, pneumonia & Fever, noticed larvae & Death (Pneumonia)** \\
\hline $26^{30}$ & 47 & м & 2012 & China & Hong Kong & Home & Tracheostomy & Manual removal & Nasopharyngeal carcinoma & Fever, discharge, noticed larvae & Stable \\
\hline $27^{88}$ & 51 & м & 2012 & China & Hong Kong & The elderly home & Limb: right big toe & Manual removal, surgical removal, antibiotics & Ulcer & Swollen, bleeding, fever, noticed larvae & Recovery/discharged \\
\hline $28^{88}$ & 84 & M & 2012 & China & Hong Kong & The elderly home & Mouth & Manual removal & Multiple underlying illnesses & Fever, noticed larvae & Stable \\
\hline $29^{88}$ & 88 & M & 2012 & China & Hong Kong & The elderly home & Mouth: upper lip, hard palate & $\mathrm{NR}$ & Multiple underlying illnesses & Noticed larvae, necrosis, swollen & Stable \\
\hline $30^{89}$ & 83 & м & 2013 & China & Hong Kong & The elderly home & Limb: left forearm & NR & Multiple underlying illnesses, bedridden, ulcer & Noticed larvae & $\mathrm{NR}$ \\
\hline $31^{89}$ & 73 & $\mathrm{~F}$ & 2013 & China & Hong Kong & The elderly home & Mouth: upper palate & Manual removal & Multiple underlying illnesses, bedridden, tube feeding & Noticed larvae & Recovery/discharged \\
\hline $32^{90}$ & 69 & м & 2013 & China & Hong Kong & The elderly home & Mouth: the hard palate & Manual removal & Multiple underlying illnesses, wheelchair bound, pneumonia & Fever, swollen, bleeding, necrosis & Death $(\mathrm{NR})^{* *}$ \\
\hline $33^{91}$ & 90 & $\mathrm{~F}$ & 2013 & China & Hong Kong & The elderly home & Mouth: gum, the upper palate & Manual removal & Multiple underlying illnesses, bedridden, tube feeding, pneumonia & Swollen, bleeding, ulcer, noticed larvae & Death (Pneumonia)** \\
\hline $34^{92}$ & 80 & $\mathrm{~F}$ & 2013 & China & Hong Kong & The elderly home & Mouth & Surgical removal & Multiple underlying illnesses, bedridden, tube feeding & Noticed larvae & Stable, discharged \\
\hline $35^{93}$ & 91 & м & 2015 & China & Hong Kong & The elderly home & Mouth & NR & Multiple underlying illnesses, bedridden, tube feeding & Feeding problem, noticed larvae, ulcer & $\begin{array}{l}\text { Death (underlying } \\
\text { illnesses) }{ }^{* *}\end{array}$ \\
\hline $36^{94}$ & 67 & M & 2015 & China & Hong Kong & RCHE & Mouth: right upper lip, palate & Manual removal & Multiple underlying illnesses, bedridden & Necrosis, bleeding, noticed larvae & Stable \\
\hline $37^{95}$ & 74 & M & 2015 & China & Hong Kong & RCHE & Mouth & Antibiotics & Multiple underlying illnesses, chest infection, bedridden & Bleeding, noticed larvae & Death $($ Pneumonia) $* *$ \\
\hline $38^{96}$ & 95 & M & 2015 & China & Hong Kong & RCHE & Mouth & NR & Multiple underlying illlnesses, tube feeding, bedridden & Ulcer, noticed larvae & Death $(\mathrm{NR})^{* *}$ \\
\hline $39^{97}$ & 67 & м & 2015 & China & Hong Kong & RCHE & $\begin{array}{l}\text { Mouth: right upper lip, upper } \\
\text { palate }\end{array}$ & Manual removal & Multiple underlying illinesses, bedridden & Necrosis, bleeding, noticed larvae, ulcer & Stable \\
\hline $40^{98}$ & 71 & M & 2015 & China & Hong Kong & RCHE & Mouth & $\mathrm{NR}$ & Multiple underlying illnesses, bedridden, chest infection & Bleeding, noticed larvae & Death (Pneumonia)** \\
\hline $44^{199}$ & 89 & $\mathrm{~F}$ & 2016 & China & Hong Kong & RCHE & Mouth & Manual removal & Multiple underlying illnesses, bedridden, tube feeding & Swollen, noticed larvae & Recovery/discharged \\
\hline
\end{tabular}




\begin{tabular}{|c|c|c|c|c|c|c|c|c|c|c|c|}
\hline $42^{99}$ & 88 & M & 2016 & China & Hong Kong & RCHE & Mouth & NR & Multiple underlying illlnesses, bedridden, tube feeding & Noticed larvae & Stable \\
\hline $43^{100}$ & 80 & M & 2016 & China & Hong Kong & NR & Limb: left ankle & Manual removal & Multiple underrying illess,s chronic leff ankle uller & Noticed larvae & Stable \\
\hline $44^{400}$ & 95 & $\mathrm{~F}$ & 2016 & China & Hong Kong & RCHE & Limb: right big toe & NR & Multiple underlyng illinss, trauma & Noticed larae & Recoverydischarged \\
\hline $45^{101}$ & 46 & $\mathrm{~F}$ & 2016 & China & Hong Kong & Home & Torso: right breast & Manual removal & Multiple underlyng illinesses, right breast wound & Noticed larvae & Recovery/discharged \\
\hline $46^{102}$ & 88 & M & 2017 & China & Hong Kong & RCHE & Mouth & Manual removal & Multiple underlying illinesses, debility & Noticed larae & Stable \\
\hline $47^{103}$ & 65 & м & 2018 & China & Hong Kong & Lov, RCHE & Mouth: palate & Manual removal, antibioitics & Infection: left dental abscess, ulcer & Fever,swollen, extensive palatal ulcers, noticed larvac & Stable, discharged \\
\hline $48^{103}$ & 83 & $\mathrm{~F}$ & 2018 & China & Hong Kong & Lov, RCHE & Mouth & Manual removal, antibioitics & Multiple undertying illinesses, arage uller & Gum bleeding, a large ulcer, noticed larvae & Death $(\mathrm{NR})^{* *}$ \\
\hline $49^{53}$ & 96 & M & 2005 & China & Hong Kong & Nursing home & Mouth & Manual removal, antibioitics, suffocating agent: turpentine & $\begin{array}{l}\text { Multiple underlying illinesses, arcinoma of the lung and prostate, dementia, } \\
\text { bedriden, infection: severe pneumonia }\end{array}$ & Fever, noticed larvae, uleer, discharge, necrosis & Death (Pneumonia) $)^{* *}$ \\
\hline $50^{53}$ & 85 & M & 2005 & China & Hong Kong & Hоmе & Face: tumour base & Manual removal, antibioitics, surffocating agent: turpentine & $\begin{array}{l}\text { Multiple underlying illinesses, stroke, gout, hypertension, leff facial basal cell } \\
\text { carcinoma, ulcer, semi-berridden }\end{array}$ & Uleer, noticed larvae & Recovery/discharged \\
\hline $51^{5^{3}}$ & 90 & $\mathrm{~F}$ & 2011 & China & Hong Kong & Nursing home & Eye & Manual removala, antibioitics, suffocating agent: turpentine & $\begin{array}{l}\text { Multiple underlying illnessese, Chronic obstructive pulmonary disease, stroke, } \\
\text { dementia, bedridden, right eyelid squamous cell carcinoma, Infection: old } \\
\text { tuberculosis and aspergillosis }\end{array}$ & Swollen, discharge, noticed larvae & $\begin{array}{l}\text { Death Mycardial } \\
\text { infarction)"w }\end{array}$ \\
\hline $52^{48}$ & 89 & $\mathrm{~F}$ & 2002 & China & Hong Kong & NR & Mouth & $\begin{array}{l}\text { Manual removala, surgeical removal, broad-spectrum } \\
\text { antibioicis sherapy }\end{array}$ & 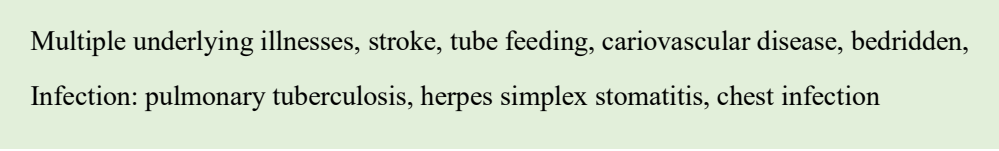 & Fever, uleer, noticed larae & 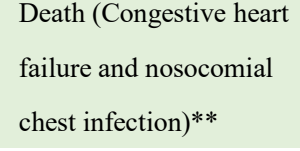 \\
\hline $53^{18}$ & 90 & $\mathrm{~F}$ & 2003 & China & Hong Kong & RCHE & $\begin{array}{l}\text { Eye: irght orbit, right upper and } \\
\text { lower eyelis }\end{array}$ & Surgical removal & 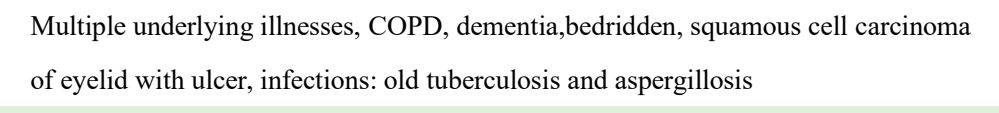 & 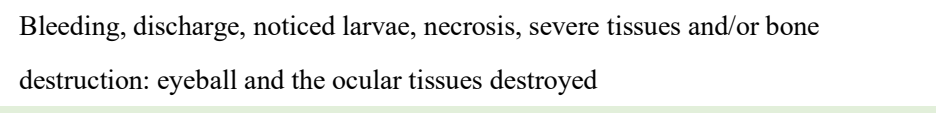 & $\begin{array}{l}\text { Deaath (COPD and } \\
\text { Myocardial infrction) }{ }^{* *}\end{array}$ \\
\hline $54^{26}$ & 89 & $\mathrm{~F}$ & 2002 & China & Hong Kong & Nursing home & Mouth: palate, jaw & Manual removal, surgical removal & Stroke, dementia, bedridden, tube feeding & Noticed larvae & Death (Pneumonia) $)^{* *}$ \\
\hline $55^{26}$ & 69 & $\mathrm{~F}$ & 2002 & China & Hong Kong & Nursing home & Mouth: palate, jaw & Manual removal, surgical removal & Strocke, demennia, bedridden, tube recting, oral ulecr & Bleeding & Recovery/discharged \\
\hline $57^{26}$ & 77 & $\mathrm{~F}$ & 2002 & China & Hong Kong & Nursing home & Mouth: palate, subclavian sinus & $\mathrm{NR}$ & Dementia, bedridden, tube feeding & Noticed larvae, bleeding & Death (Pneumonia)** \\
\hline $58^{26}$ & 79 & $\mathrm{~F}$ & 2003 & China & Hong Kong & Nursing home & $\begin{array}{l}\text { Perineal \& inguina legeions: } \\
\text { labia majora }\end{array}$ & Manual removal & Hypertension, dementia, bedriden, tube feeding, wound: Sacral bed Sore & Noticed larvae & Recovery/discharged \\
\hline $59^{26}$ & 71 & M & 2003 & China & Hong Kong & Nursing home & Limb: foot & $\begin{array}{l}\text { Manul removal, antibioicis, surgical removal and toe } \\
\text { amputation }\end{array}$ & Diabetes mellitus, diabetic foot uler, blindness & $\begin{array}{l}\text { Fevere, discharge, foul smell, noticed larva, severe tissuses and dor bone } \\
\text { destruction }\end{array}$ & Recovery/discharged \\
\hline $60^{26}$ & 96 & м & 2003 & China & Hong Kong & Nursing home & $\begin{array}{l}\text { Mouth: hard palate, maxillary } \\
\text { sinuses }\end{array}$ & Manual removal, antibioitics & Carcinoma of the prostate, dementia, bedrididen & Fever, secondary ynfection & Death (Pneumonia)*** \\
\hline $61^{104}$ & 90 & $\mathrm{~F}$ & 2004 & China & Hong Kong & The elderly home & Mouth & Manual removal, surgical removal, antibiotics & $\begin{array}{l}\text { Multiple underlying illinesses, bedridden, tube feeding, diabetes mellitus, } \\
\text { hyperension, dementia and haemorragaic stroke with limb contracturs, bacteral } \\
\text { infection }\end{array}$ & Fever, swollen, blededing, noticed laraa, necrosis & Recovery \\
\hline $62^{105}$ & 68 & м & 2005 & China & Hong Kong & $\begin{array}{l}\text { A street leeper, poor, } \\
\text { low }\end{array}$ & Limb: the right ankle & Suffocating gagnt: turpentine, surgical removal & Uler & Ulcer, bleeding, noticed larvae & Recovery/discharged \\
\hline $63^{105}$ & 90 & $\mathrm{~F}$ & 2005 & China & Hong Kong & $\mathrm{NR}$ & Mouth & Manual removal, surgical removal, antibioitics & Mental illness, twbe feeding, bedridden & Ulcer, noticed larvae, necrosis & Recovery/discharged \\
\hline $64^{106}$ & 86 & $\mathrm{~F}$ & 2014 & China & Hong Kong & Home, rural & Limb: leff foot & Manual removal, surgical removal, antibiotics & Leff foot erythema, ulcer, infection & Noticed larvae & Recovery \\
\hline $65^{106}$ & 74 & M & 2014 & China & Hong Kong & Rural & Limb: right foot & Manual removal, surgical removal, antibiotics & End-sage renal disiease, right foot trauma, bacterial infections & Noticed larvae & Recovery \\
\hline $66^{106}$ & 43 & м & 2014 & China & Hong Kong & Low & Limb & Manual removal, surgical removal & 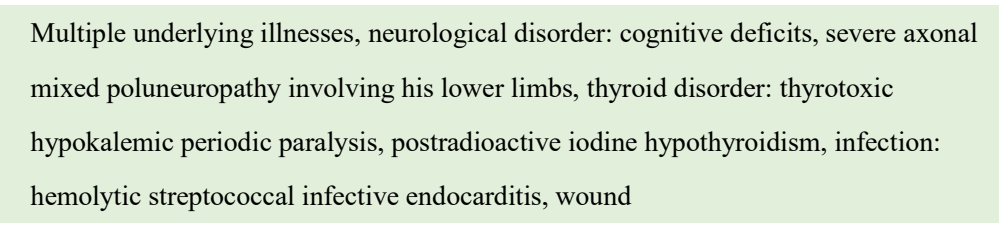 & Noticed larvae, necrosis & Recovery/discharged \\
\hline $67^{106}$ & 54 & M & 2014 & China & Hong Kong & Low & Limb: left lower shin & Manual removal, surgical removal, antibiotics, & Chronic dermatitis & Noticed larvae, necrosis & Recovery/discharged \\
\hline $68^{37}$ & 61 & $\mathrm{~F}$ & 2004 & China & Hong Kong & $\mathrm{NR}$ & Torso: right breast & Manual removal, surgical removal & Invasive lobular carcinoma & Unberabble pain, foul smell, necrosis, ulcer, discharge, blecding, noticed larvaa & Recovery/discharged \\
\hline $87^{14}$ & 2 & M & 1980 & China & $\begin{array}{l}\text { Guixian, } \\
\text { Guangxi }\end{array}$ & Low, rural & Ear & NR & Ear infection & Discharge, noticed larvac & Recovery/discharged \\
\hline $88^{14}$ & 6 & $\mathrm{~F}$ & 1980 & China & $\begin{array}{l}\text { Tianyang, } \\
\text { Guangexi }\end{array}$ & Low, nural & Ear & NR & Ear infection & Noticed larvae & Recovery/discharged \\
\hline $89^{14}$ & 37 & $\mathrm{~F}$ & 1979 & China & Daxin, Guangxi & Low, rural & Perineal \& inguinal regions & NR & Infection: vulvitis and vaginis & Tunnels, honeycomb-like both side of the labia majora, noticed larrac & Recovery/discharged \\
\hline $90^{14}$ & 49 & $\mathrm{~F}$ & 1978 & China & $\begin{array}{l}\text { Guiping, } \\
\text { Guangxi }\end{array}$ & Low, rural & Nose & NR & Infection: atrophic rhinitis & Bleeding: thinorrhagia, cold, fever, headache, noticed larae & Recovery/discharged \\
\hline $91^{14}$ & 41 & $\mathrm{~F}$ & 1973 & China & Yining, Guangxi & Low, rural & $\begin{array}{l}\text { Perineal \& inguinal regions: the } \\
\text { right of anus }\end{array}$ & $\mathrm{NR}$ & Miscarriage & Tunnels & Recovery/discharged \\
\hline $92^{14}$ & 1 & м & 1966 & China & $\begin{array}{l}\text { Nanning, } \\
\text { Guangxi }\end{array}$ & City & Ear: left ear canal & NR & Ear infection & Noticed larvae & Recovery/discharged \\
\hline $93^{14}$ & 8 & M & 1959 & China & $\begin{array}{l}\text { Pinxiang, } \\
\text { Guangxi }\end{array}$ & City & Nose & NR & Infection: thinitis & Fever, discharge, foul smell, tumels & Recovery/discharged \\
\hline $94^{42}$ & 56 & $\mathrm{~F}$ & 1958 & China & $\begin{array}{l}\text { Nanning, } \\
\text { Guangxi }\end{array}$ & Low, rural & Nose & Manual removal & Infection: thinitis & Fever, discharge, foul Imell, headache, uleer, noticed larvae & Recovery/discharged \\
\hline $95^{2}$ & NR & NR & 1970 & China & Yuman & NR & Nose & NR & $\mathrm{NR}$ & NR & Recovery/discharged \\
\hline $96^{\circ}$ & 84 & F & 2009 & China & $\begin{array}{c}\text { Lufeng, } \\
\text { Guangdong }\end{array}$ & Low, rural & Limb & Manual removal, suffocating agent & Hypertension, stroke & $\begin{array}{l}\text { Large ulerer, severe pain, discharge, inflammation, odedema, sensation of moving } \\
\text { larrac, noticed larva, pruritus }\end{array}$ & Recovery/discharged \\
\hline $97^{73}$ & 54 & $\mathrm{~F}$ & 2007 & China & Jiangxi & Low, rural & Mouth, Nose & Manual removal, anitiboitis & № & $\begin{array}{l}\text { Huge ulcer between the hard and soft palate, connected with nose, necrosis, } \\
\text { intens pe pain, eating difficulty, blecding }\end{array}$ & Recovery/discharged \\
\hline
\end{tabular}




\begin{tabular}{|c|c|c|c|c|c|c|c|c|c|c|c|}
\hline $98^{61}$ & 49 & $\mathrm{~F}$ & 1981 & China & $\begin{array}{l}\text { Quanzhou, } \\
\text { Fujian }\end{array}$ & Low, rural & Perineal \& inguinal regions & Surgical removal & NR & Pruritus vulvae, pain, discharge, edema, tunnels, noticed larvae & Recovery/discharged \\
\hline $99^{64}$ & 56 & M & 2015 & China & $\begin{array}{l}\text { Wei County, } \\
\text { Hebei }\end{array}$ & Low, rural & Mouth & Manual removal & № & Pain, edema, redness, necrosis, ulcer, noticed larvae & Recovery/discharged \\
\hline $100^{52}$ & 45 & $\mathrm{~F}$ & 2006 & India & New Delhi & Low & Perineal \& inguinal regions & Manual removal, suffocating agent: turpentine & Carcinoma cervix, bladder incontinence, ulcer & Pruritus, pain, noticed larvae & Discharged \\
\hline $101^{108}$ & 22 & M & 2014 & India & Delhi & Low & Mouth & $\begin{array}{l}\text { Manual removal, suffocating agent, antibiotics, } \\
\text { insecticides: ivermectin }\end{array}$ & $\begin{array}{l}\text { Neurological disorder and mental illness: cerebral plasy, severe mental retardation, } \\
\text { quadriplegia, and kyphoscoliosis, seizure disorder }\end{array}$ & Noticed larvae, ulcer, swollen & Recovery/discharged \\
\hline $102^{109}$ & 71 & $\mathrm{NR}$ & 1990 & India & Dellii & Low & Eye & Manual removal & Infection: herpes zoster opththalmicus on the face & NR & NR \\
\hline $103^{56}$ & 65 & M & 2016 & India & Chhattisgarh & Low & Right eye & $\begin{array}{l}\text { Manual removal, surgical removal, suffocating agent: } \\
\text { turpentine oil, antibiotics, insecticides: ivermectin }\end{array}$ & Debility, immunocompromised, an empty orbital socket postevisceration & Pain, redness, discharge, swollen, necrosis, ulcer & Recovery/discharged \\
\hline $104^{38}$ & 65 & $\mathrm{~F}$ & 1994 & India & Shertallai & Low & Limb: left foot & Antibiotics & Infection:filarial, lymphoedema with dermatosclerosis and papillomatosis, ulcer & Fever, chill, pain, discharge, noticed larvae & Recovery \\
\hline $105^{38}$ & 60 & M & 1994 & India & Shertallai & Low & Limb: eff leg & Manual removal, suffocating agent: turpentine, antibiotics & Infection:filarial, lymphoedema with ulcers in both legs & Fever, chill, discharge & Recovery \\
\hline $106^{38}$ & 33 & $\mathrm{M}$ & 1994 & India & Pondicherry & Low & Limb: left ankle & Manual removal, antibiotics & Infection:filarial lymphoodema in the left leg, ulcer & Severe pain, reddish, noticed larvae & Recovery \\
\hline $107^{110}$ & 60 & M & 2011 & India & Punjub & Low & Mouth & Manual removal & Squamous cell carcinoma in oral cavity, ulcer, perforation of cheek & Severe pain, foul smell, discharge, noticed larvae & Recovery \\
\hline $108^{110}$ & 32 & $\mathrm{~F}$ & 2011 & India & Punjab & Low & Face, mouth & $\begin{array}{l}\text { Manual removal, surgical removal, suffocating agent: } \\
\text { turpentine }\end{array}$ & Squamous cell carcinoma in oral cavity, ulcer, perforation of cheek & Swollen, noticed larvae & Recovery \\
\hline $109^{27}$ & 50 & $\mathrm{~F}$ & 2005 & India & Punjub & Low & Limb & NR & Diabetes mellitus, diabetic foot, trauma, numbness & Noticed larvae & NR \\
\hline $110^{27}$ & 30 & M & 2006 & India & Patiala & Low & Limb & Surgical removal, left foot amputation & Trauma, diabetes mellitus, diabetic foot, ulcer & $\begin{array}{l}\text { High fever, discharge, noticed larvae, severe tissues and/or bone destruction: a } \\
\text { bone deep gangrenous wound, necrosis }\end{array}$ & NR \\
\hline $111^{51}$ & 12 & M & 2012 & India & $\mathrm{NR}$ & Low & Mouth & $\begin{array}{l}\text { Manual removal, suffocating agent: cotton bud } \\
\text { impregnated with turpentine }\end{array}$ & Neurological disorder & Swollen, tunnels, noticed larvae, foul smell & Recovery \\
\hline $112^{111}$ & 22 & M & 2011 & India & Telangana & Low & Mouth & Manual removal & Mental illness & Pain, irritataion, distress, bleeding, inflammation, abscess, ulcer & Recovery/discharged \\
\hline $113^{111}$ & 90 & F & 2011 & India & Telangana & Low & Nose & Manual removal, suffocating agent & Hypertension, diabetes mellitus & Pain, distress, edema & Recovery/discharged \\
\hline $114^{112}$ & 4 & $\mathrm{~F}$ & 2014 & India & Madhya Pradesh & Low & $\begin{array}{l}\text { Perineal \& inguinal regions: } \\
\text { Prolapsed rectum }\end{array}$ & Manual removal, suffocating agent & NR & Noticed larvae, ulceration,fever, inflammation & Recovery/discharged \\
\hline $115^{31}$ & 73 & M & 2013 & India & Chennai & $\mathrm{NR}$ & Tracheostomy & Manual removal & Diabetes mellitus, carcinoma supraglottis, tracheostomy & Bleeding, discharge, ulcer, noticed larvaa, edema, foul smell & Recovery/discharged \\
\hline $116^{113}$ & 33 & M & 2010 & India & West Bengal & Low & Perineal \& inguinal regions & Manual removal, antibiotics & Dermatitis, giant perigenital lesion, ulcer, mental illness & Noticed larvae, discharge & NR \\
\hline $117^{50}$ & 42 & $\mathrm{~F}$ & 2015 & India & Assam & Low & Eye: right eye & $\begin{array}{l}\text { Manual removal, surgical removal, suffocating agent: } \\
\text { turpentine, antibiotics }\end{array}$ & Trauma & Swollen, itch, bleeding, foul smell, discharge, noticed larvae & Recovery/discharged \\
\hline $118^{114}$ & 12 & $\mathrm{~F}$ & 2009 & India & Madhya Pradesh & NR & Mouth: gum & Manual removal, surgical removal, antibiotics & Gingival lesion, ulcer & Pain, swollen, bleeding, noticed larvae & Recovery \\
\hline $119^{115}$ & 70 & $\mathrm{~F}$ & 2014 & India & $\mathrm{NR}$ & Low & Mouth: anterior maxilla & $\begin{array}{l}\text { Manual removal, surgical removal, suffocating agent: } \\
\text { turpentine, antibitics }\end{array}$ & Malnutrition & Swollen, pain, necrosis, severe tissues and/or bone destruction & Recovery \\
\hline $120^{57}$ & 12 & м & 2010 & India & NR & Low & Mouth & $\begin{array}{l}\text { Manual removal, suffocating agent: turpentine, } \\
\text { insecticides: ivermectin }\end{array}$ & $\begin{array}{l}\text { Neurological disorder: seizures, unconscious state, malnutrition, debility, tube } \\
\text { feeding, ulcer, mouth breathing, incompetent lips }\end{array}$ & Swollen, reddish, tunnels, noticed larvae & Recovery/discharged \\
\hline $121^{116}$ & 40 & M & 2013 & India & NR & Low & Mouth & $\begin{array}{l}\text { Manual removal, surgical removal, insecticides: } \\
\text { ivermectin, antibiotics }\end{array}$ & NR & Pain, swollen, noticed larvae, inflammation, fever, bone loss & Recovery/discharged \\
\hline $122^{117}$ & 14 & M & 2010 & India & $\mathrm{NR}$ & Low & Mouth & Manual removal, suffocating agent: turpentine, antibiotics & Mental illness, neurological disorder: seizure, ulcer & Swollen, severe pain, fever, apprehensive, necrosis, noticed larvae & Recovery/discharged \\
\hline $123^{118}$ & 51 & $\mathrm{~F}$ & 2013 & India & $\mathrm{NR}$ & Low & Mouth & $\begin{array}{l}\text { Manual removal, surgical removal, suffocating agent: } \\
\text { turpentine, antibiotics }\end{array}$ & $\begin{array}{l}\text { Infection: filarial lymphedema and elephantiasis, lepross, incompetent lips, advanced } \\
\text { periodontal disease }\end{array}$ & $\begin{array}{l}\text { Pain, sensation of moving larvae, noticed larvae, inflammation, discharge, } \\
\text { tunnels, bone destruction in teeth }\end{array}$ & Recovery/discharged \\
\hline $124^{54}$ & 35 & $\mathrm{~F}$ & 2012 & India & NR & Low & Mouth & $\begin{array}{l}\text { Manual removal, surgical removal, suffocating agent: } \\
\text { cottons buds impregnated with turpentine oil, insecticides: } \\
\text { ivermectin, antibiotics }\end{array}$ & Mental illness, mouth breathing & Severe pain, swollen, noticed larvae, foul smell, tunnels & Recovery/discharged \\
\hline $125^{32}$ & 46 & $\mathrm{~F}$ & 2008 & India & Ariankuppam & Low & Tracheostomy & Manual removal, suffocating agent: turpentine & Advanced carcinoma of hypopharynx and neck, tracheostomy & Noticed larvae & $\mathrm{NR}$ \\
\hline $126^{32}$ & 52 & $\mathrm{~F}$ & 2008 & India & Tamil Nadu & Low & Tracheostomy & Manual removal, surgical removal, antibiotics & Carcinoma of larynx, tracheostomy, bacterial infections & Noticed larvae & Discharged \\
\hline $127^{32}$ & 62 & M & 2008 & India & Tamil Nadu & Low & Face & NR & Carcinoma of cheek & Discharge, foul smell & NR \\
\hline $128^{32}$ & 75 & M & 2008 & India & Tamil Nadu & Low & Mouth & $\mathrm{NR}$ & Ulcer, lip cancer & Bleeding, discharge, noticed larvae & NR \\
\hline $129^{16}$ & 30 & M & 1991 & India & NR & Low & Limb: lower left leg & Manual removal & Ulcer, trauma, drug addict (heroin), infection: active cellulitis & $\begin{array}{l}\text { Mixed infestation by Chrysomya bezziana and Sarcophaga sp., severe pain, } \\
\text { noticed larvae, foul smell, discharge, severe tissues and } / / \text { bone destruction: a } \\
\text { bone-beep non-healing ulcer with } 10 \mathrm{~cm} \times 10 \mathrm{~cm} \text { in size }\end{array}$ & NR \\
\hline $130^{119}$ & 14 & M & 2011 & India & $\mathrm{NR}$ & Low & Perineal \& inguinal regions & Manual removal, antibiotics, suffocating agent: paraffin & Infections: perianal codylomata acuminata, bacterial infections & Pain, discharge, itch, foul smell, bleeding, necrosis, noticed larvae & NR \\
\hline $131^{120}$ & 32 & $\mathrm{~F}$ & 2011 & India & $\mathrm{NR}$ & Low & Mouth: hard palate and gums & Manual removal, insecticides: ivermectin, surgical removal & Mental illness, mouth breathing & Extensive necrosis, bleeding, foul smell, pain, swollen & Recovery/discharged \\
\hline $132^{121}$ & - & - & 1971 & India & - & & Mouth & - & - & - & - \\
\hline $133^{33}$ & 78 & M & 2011 & India & NR & Low & Tracheostomy & $\begin{array}{l}\text { Manual removal, surgical removal, suffocating agent: } \\
\text { turpentine soaked gauze (but need caution while using it } \\
\text { around the tracheostoma, a high risk of chemical } \\
\text { pneumonitis), antibiotics }\end{array}$ & Head injury, trauma, tracheostomy, respiratory distress, bacterial infections & Foul smell, bleeding, discharge, noticed larvae & Recovery/discharged \\
\hline $134^{19}$ & 80 & $\mathrm{~F}$ & 1990 & India & NR & Low & Eye & Manual removal,suffocating agent: turpentine, antibiotics & No & $\begin{array}{l}\text { Bleeding, discharge, noticed larvae, inflammation, ulcer, foul smell, severe } \\
\text { tissues and/or bone destruction: complete loss globe in two days }\end{array}$ & Recovery/discharged \\
\hline $135^{46}$ & 50 & $\mathrm{~F}$ & 2018 & India & $\mathrm{NR}$ & Rural, low & Nose & Manual removal, suffocating agent, antibiotic & Diabetes mellitus, nasal obstruction and pisitaxis & $\begin{array}{l}\text { Noticed larvae, bleeding, necrosis, discharge, tunnels, severe tissues and/or bone } \\
\text { destruction: destroy of bony septum }\end{array}$ & Recovery/discharged \\
\hline $136^{5}$ & 35 & $\mathrm{~F}$ & 1909 & India & Assam & Low & Nose, eye, face, mouth & $\begin{array}{l}\text { Thousands of screw-worms with several generations were } \\
\text { found, removed by manual removal (including nose, eye, } \\
\text { face and mouth), using suffocating agent(nose): turpentine, } \\
\text { treatment: nasal douche }\end{array}$ & $\begin{array}{l}\text { Multiple underlying illnesses, Infections: ankylostomiasis, malaria, bronchitis, } \\
\text { anaemia, asthma }\end{array}$ & $\begin{array}{l}\text { Noticed larvae, fever, acute inflammation, Tunnels,oedema, bleeding, foul } \\
\text { smell, discharge, pain, necrosis, severe tissues and/or bone destruction: from } \\
\text { below the nostrils to halfway up to forehead. eyelids and cheeks, including } \\
\text { subcutaneous tissue, muscles, mucous membrane, cartiliage, periosteum and } \\
\text { even bone }\end{array}$ & $\begin{array}{l}\text { Death (exhaustion with } \\
\text { rapid and heavy tissue } \\
\text { destruction caused by a } \\
\text { large number of maggots, } \\
\text { and other underlying }\end{array}$ \\
\hline
\end{tabular}


Surgical removal, manual removal, suffocating agent:

Mouth: the posterior region of

$137^{55}$

$139^{19}$

$140^{34}$

$141^{20}$

\section{NR NR}

Ind

F $1990 \quad$ In

$55 \quad$ F 20

$87 \quad \mathrm{~F} \quad 20$

$87 \quad$ M 2009

$18 \quad$ M 2009

$5 \quad$ M 2007

F 2015

F 2000

$1993 \quad$ Ir

$\begin{array}{llll}65 & \text { F } & 2009 & \text { Ir }\end{array}$

$65 \quad$ M 2002

$55 \quad$ F $1997 \quad$ Sri Lanka

$73 \quad$ M $\quad 1997 \quad$ Sri Lanka

$60 \quad$ M $\quad 1997 \quad$ Sri Lanka

$49 \quad$ M $\quad 1997 \quad$ Sri Lank

$39 \quad$ M $\quad 1997 \quad$ Sri Lanka

$\begin{array}{llll}65 & \text { F } & 1997 & \text { Sri Lanka }\end{array}$

$42 \quad$ M $\quad 1997 \quad$ Sri Lanka

$\begin{array}{llll}73 & \text { M } & 1997 & \text { Sri Lanka }\end{array}$

$1997 \quad$ Sri Lanka

76 M 2016-2017 Sri Lanka

Perak

Low

NR NR

NR Low

NR NR

Colombo Low

Colombo Low

Colombo Low

Colombo Low

Colombo Low

Colombo Low

Colombo Low

Colombo Low

Colombo low

Colombo Low

Colombo Low

Colombo Low

Colombo Low

Colombo Low
89 M $2012 \quad$ Ir

$94 \quad$ M $\quad 1997 \quad$ Sri Lanka the jaw

Scalp

Eye: globe

Pharyngostomy

Eye: left ey

Ear

Eye: left orbi

Limb

Mouth

Scalp

Eye

Perineal \& inguinal regions Manual removal

Manual removal, antibiotics

Mouth

Face, nose, nosal sinuses,

mouth: upper lip, eye: left orbit

Limb: right big toe

Saunsar village Low, rural

Island offshore
of Langkawi

Renideniya,

insecticides: ivermectin

antibiotics turpentine

Surgical removal, antibiotics

Surgical removal

Manual removal

Surgical removal

Surgical removal, antibiotics big toe

\section{Perineal \& inguinal regions}

Perineal \& inguinal region

vagin

Scalp

Limb: fourth toe, right foc

Limb: right heel

Limb: right tig toe

Scalp

\section{Limb: dorsum of left foot}

Limb: right foot

Limb: leff foot

Limb: foot

Limb: foot

Limb: foot

Limb: foot$$
\text { NR }
$$

$\mathrm{NR}$

b: right heel
Manual removal, suffocating agent: turpentine linimen

Manual removal, surgical removal, suffocating agent:

Surgical removal, suffocating agent:

chloroform-containing sterile oil, antibiotics

Manual removal, surgical removal, antibiotics

Manual removal, surgical removal and orbital exenteration Leukomatous lesion, the eyelid basal cell carcinoma, chronic eye infection

Manual removal, surgical removal and amputation of the Diabetic mellitus, diabetic foot ulcer
Manual removal, antibiotics: amoxicillin
Manual removal, suffocating agent: a pack soaked with turpentine oil kept in vagina, antibiotics

Manual removal, antibiotics

Manual removal, suffocating agent: oil, plugging the nose with dextrose solution

Surgical removal, Manual removal, wound dressed with $\quad$ Infection: otitis media chronica, conductive and nerve deafness, cholesteatomatous plugs soaked in 10\% dextrose solution (dress every day) cavily

Manual removal, suffocating agent: turpentine

Manual removal, suffocating agent: turpentine

Manual removal, suffocating agent: turpentine

Manual removal, suffocating agent: turpentine

Manual removal, suffocating agent: turpentine

Manual removal, suffocating agent: turpentine

Manual removal, suffocating agent: turpentine

Manual removal, suffocating agent: turpentine

Manual removal, suffocating agent: turpentine

Manual removal, suffocating agent: turpentine

Manual removal, suffocating agent: turpentine

Manual removal, suffocating agent: turpentine

Manual removal, suffocating agent: turpentine

Manual removal, suffocating agent: turpentine

Manual removal

Mental illness

HIV and hepatitis virus infections, immunocompromised state, wound: lef

mediolateral episiotomy wound postnatal period

Chronic seborrhoeic eczen

fection: sinusitis and rhinitis atrophicans, anem

Dermatitis, mental illness

Dermatitis

Dermatitis

Infection: scalp sepsis, ectoparasite: pediculosis, mental illness: mental retardation

Dermatitis

Ulcer, infections: leprosy, dermatitis

Dermatitis, senile debility

Ulcer

Diabetes mellitus, diabetic ulcer

Trauma

Mental illness

NR

NR

Diabetes mellitus, diabetic ulcer
Infection: appendicitis

Multiple underlying illnesses, non-Hodgkin lymphoma, dementia, infection: sepsis immunocompromised

Facial squamous cell carcinoma, wound

(1)

gested aluminum hydroxide powder by accident, severe burns of oral cavity and esophagus, pharyngostomy tube

Prolonged ear infection

Wultiple underlying illnesses, trauma, cancer, eyelid surgical removal due to skin nn, back injury, bladder and bowel incontinence

ultiple underlying illnesses, diabetes mellitus, diabetic feet uleers, cardiovascular

mental retardation, severe failure to thrive, oral lesion, ulcer

Carcinoma of the rectum, ulcer

High fever, severe pain, noticed larvac

Recovery/discharged

A large ulcer of about $6 \mathrm{~m} \times 2.5 \mathrm{~cm}$, inflammation, bleeding, discharge, foul

mell, Severe tissues and/or bone destruction: the globe completely destroyed in Discharged

Noticed larvae, swollen

Stable

Noticed larvae, pain, severe tissues and/or bone destruction: left eye completely destroyed

Pain, dischar

Recovery/discharged

Severe pain and necrosis, noticed larvae, swollen, redness, severe tissues and/or $\quad$ Recovery/discharged

bone destruction: eyelids and eyeball completely destroyed

Severe itch

NR

Pain, noticed larvae

Recovery/discharged

Severe headache, agitation, severe tissue destruction, wound, noticed larvae

Death (underlying

diseases worsen, severe

epsis, septic shock,

multiorgan failure)**

Severe tissue/bone destruction: ocluar rupture and larvae penetrate deep enough

to involve the optic canal, noticed larvae, severe swollen, discharge, bleeding

Severe abdominal pain, dysuria, noticed larvac

Recovery/discharged

Fever, necrosis, noticed larvac

Death (Sepsis)**

Bleeding, pain, discharge, noticed larvae, ulcer, severe tissues and/or bone

destruction: soft tissues malignancy involving left orbit, nose, maxillary sinus $\quad$ Discharged

and sphenoid bone

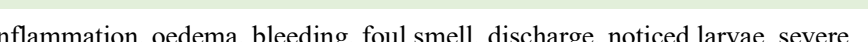
iissues and/or bone destruction

Bleeding: epistaxis, headache, noticed larvae

Foul smell

Pain, bleeding, discharge, noticed larvae, sensation of moving larvac,

Recovery/discharged

Pain, irritation, edema, wound, noticed larvae

Recoverydischarged

Pain, bleeding, noticed larvae, ulceration, sensation of moving larvae, itch Recovery/discharged

Discharge, foul smell, headache, bleeding, fever, edema, severe tissue and/or $\quad$ Recoveryddischarged

bone destruction: septum defect, noticed larvac

Recovery/discharged

Discharge, foul smell, bone destruction, pain

Discharged

Discharged

Discharged

Discharged

Discharged

Discharged

Discharged

Discharged

Discharged

Discharged

Discharged

Discharged

Discharged

Discharged

Discharged 


\begin{tabular}{|c|c|c|c|c|c|c|c|c|c|c|c|}
\hline $176^{37}$ & 30 & M & 2016-2017 & Sri Lanka & Kandy city, KA & Middle class & Limb: right heel & Manual removal & Diabetes mellitus, diabetic uler & NR & Discharged \\
\hline $177^{37}$ & 65 & $\mathrm{~F}$ & 2016-2017 & Sri Lanka & Thalathuoya, KA & Low, poor, rural area & Limb: left leg 2nd digit & Manual removal & Wound & NR & Discharged \\
\hline $178^{37}$ & 92 & M & 2016-2017 & Sri Lanka & $\begin{array}{c}\text { Muruthalawa, } \\
\text { KA }\end{array}$ & Low, poor, rural area & Limb: right ankle & Manual removal & Mental illness, wound & $\mathrm{NR}$ & Discharged \\
\hline $179^{37}$ & 39 & M & $2016-2017$ & Sri Lanka & $\begin{array}{l}\text { Wuraganthota, } \\
\text { KA }\end{array}$ & Low, poor, rural area & Limb: left calf & Manual removal & Infection: filarial lymphedema, wound & NR & Discharged \\
\hline $180^{37}$ & 65 & M & 2016-2017 & Sri Lanka & Galaha,KA & Low, poor, rural area & Torso: left buttock & Manual removal & Mental illness, wound & $\mathrm{NR}$ & Discharged \\
\hline $181^{37}$ & 54 & $\mathrm{~F}$ & 2016-2017 & Sri Lanka & Meepalawa,KA & Low, poor, rural area & Limb: right heel & Manual removal & Diabetes mellitus, diabetic uler & $\mathrm{NR}$ & Discharged \\
\hline $182^{37}$ & 39 & M & 2016-2017 & Sri Lanka & $\begin{array}{l}\text { Hakgala,BA } \\
\text { (Badulla) }\end{array}$ & Low, poor, rural area & Limb: right foot & Manual removal & Diabetes mellitus, diabetic ulcer & NR & Discharged \\
\hline $183^{37}$ & 70 & $\mathrm{~F}$ & $2016-2017$ & Sri Lanka & $\begin{array}{l}\text { Agarapathana, } \\
\text { NU (Nuwara } \\
\text { eliya) }\end{array}$ & Low, poor, rural area & Limb: left calf & Manual removal & Wound & NR & Discharged \\
\hline $184^{37}$ & 70 & M & $2016-2017$ & Sri Lanka & $\begin{array}{l}\text { Molagoda, KE } \\
\text { (Keyalle) }\end{array}$ & Low, poor, rural area & Limb: right heel & Manual removal & Diabetes mellitus, diabetic ulcer & NR & Discharged \\
\hline $185^{37}$ & 79 & M & 2016-2017 & Sri Lanka & Heeloya,BA & Low, poor, rural area & $\begin{array}{l}\text { Perineal \& inguinal regions: } \\
\text { urogenital tract }\end{array}$ & Manual removal & Mental illness & NR & Discharged \\
\hline $186^{37}$ & 75 & $\mathrm{~F}$ & 2016-2017 & Sri Lanka & Kandy city, KA & Middle class & Limb: left great toe & Manual removal & Diabetes mellitus, diabetic ulcer & NR & Discharged \\
\hline $187^{37}$ & 69 & M & 2016-2017 & Sri Lanka & Poonagala, BA & Low, poor, rural area & Limb: right foot & Manual removal & Diabetes mellitus, diabetic ulcer & NR & Discharged \\
\hline $188^{37}$ & 52 & M & 2016-2017 & Sri Lanka & $\begin{array}{l}\text { Dewaragampola, } \\
\mathrm{KE}\end{array}$ & Low, poor, rural area & Limb: left ankle & Manual removal & Diabetes mellitus, diabetic ulcer & NR & Discharged \\
\hline $189^{37}$ & 64 & M & 2016-2017 & Sri Lanka & Pallepola,KA & Low, poor, rural area & Limb: light ankle & Manual removal & Mental illness, wound & NR & Discharged \\
\hline $190^{37}$ & 55 & M & 2016-2017 & Sri Lanka & Owatta,KE & Low, poor, rural area & Limb: left ankle & Manual removal & Diabetes mellitus, diabetic uler & NR & Discharged \\
\hline $191^{37}$ & 53 & M & 2016-2017 & Sri Lanka & Nayawela,KE & Low, poor, rural area & Limb: right leg 2-3 digits & Manual removal & Diabetes mellitus, diabetic uler & NR & Discharged \\
\hline $192^{37}$ & 61 & M & 2016-2017 & Sri Lanka & $\begin{array}{c}\text { Mawanella town, } \\
\mathrm{KE}\end{array}$ & Middle class & Limb: left ankle & Manual removal & Diabetes mellitus, diabetic ulcer & NR & Discharged \\
\hline $193^{37}$ & 64 & M & 2016-2017 & Sri Lanka & Owaththa, KE & Low, poor, rural area & Limb: right calf & Manual removal & Diabetes mellitus, diabetic ulcer & $\mathrm{NR}$ & Discharged \\
\hline $194^{37}$ & 57 & $\mathrm{~F}$ & 2016-2017 & Sri Lanka & Nayawela,KE & Low, poor, rural area & Torso: right buttock & Manual removal & Diabetes mellitus, diabetic uler & NR & Discharged \\
\hline $195^{37}$ & 45 & $\mathrm{~F}$ & 2016-2017 & Sri Lanka & $\begin{array}{l}\text { Uthuwankanda, } \\
\qquad \mathrm{KE}\end{array}$ & Low, poor, rural area & Limb: right ankle & Manual removal & Diabetes mellitus, diabetic uler & NR & Discharged \\
\hline $196^{37}$ & 45 & $\mathrm{~F}$ & 2016-2017 & Sri Lanka & Aranayake, $\mathrm{KE}$ & Low, poor, rural area & Scalp & Manual removal & Mental illness, wound & NR & Discharged \\
\hline $197^{37}$ & 48 & $\mathrm{~F}$ & 2016-2017 & Sri Lanka & Medawala, KA & Low, poor, rural area & Limb: right calf & Manual removal & Infection: flalarial lymphedema, wound & NR & Discharged \\
\hline $198^{37}$ & 51 & м & 2016-2017 & Sri Lanka & $\begin{array}{c}\text { Nawalapitiya, } \\
\text { KA }\end{array}$ & Low, poor, rural area & Torso: umbilical & Manual removal & Diabetes mellitus & NR & Discharged \\
\hline $199^{37}$ & 64 & M & 2016-2017 & Sri Lanka & Pujapitiya,KA & Low, poor, rural area & Limb: right ankle & Manual removal & Diabetes mellitus, wound & NR & Discharged \\
\hline $200^{37}$ & 72 & $\mathrm{~F}$ & 2016-2017 & Sri Lanka & Galagedara,KA & Low, poor, rural area & Limb: left ankle & Manual removal & Diabetes mellitus, diabetic uler & NR & Discharged \\
\hline $201^{37}$ & 51 & M & 2016-2017 & Sri Lanka & Aranayake,KA & Low, poor, rural area & Limb: right great toe & Manual removal & Wound & NR & Discharged \\
\hline $202^{132}$ & 55 & $\mathrm{~F}$ & 2019 & Indonesia & $\mathrm{NR}$ & Low & Nose, eye & Surgical removal, manual removal, antibiotics & $\begin{array}{l}\text { Diabetes mellitus, diabetic neuropathy, infection: pansinusitis and right } \\
\text { pleuropneumonia }\end{array}$ & $\begin{array}{l}\text { Necrosis, ulcer, edema, severe tissues and/or bone destruction: destroy of nasal } \\
\text { septum and bone, abscess }\end{array}$ & Discharged \\
\hline $203^{49}$ & 9 & M & 2008 & Indonesia & Kalimantan & $\mathrm{NR}$ & Eye, ear & Surgical removal & Ear infection & Bleeding, discharge, noticed larvae, inflammation, wound & Recovery/discharged \\
\hline $204^{133}$ & 72 & M & 2009 & Laos & Luang Namtha & Low & Limb: left lower leg & Surgical removal, antibiotics & Infections: chromoblastomycosis, cellulitiss, leprosy, ectoparasite: leech bites, lesions & Noticed larvae, discharge, swollen, pain, ulcer & Recovery/discharged \\
\hline $205^{134}$ & 5 & $\mathrm{~F}$ & 2009 & Pakistan & Peshawar & Poor, low & $\begin{array}{l}\text { Perineal \& inguinal regions: } \\
\text { urogenital tract }\end{array}$ & $\mathrm{NR}$ & $\mathrm{NR}$ & Pain, viginal itch, dysuria, noticed larvae on the bed, discharge, ulcer & Recovery/discharged \\
\hline $206^{135}$ & - & - & 1959 & DRC & - & - & - & - & - & - & - \\
\hline $207^{135}$ & - & - & 1959 & DRC & - & - & - & - & - & - & - \\
\hline $208-215^{+}$ & $\mathrm{NR}$ & NR & 2007,2008 & Yemen & $\mathrm{NR}$ & NR & $\mathrm{NR}$ & $\mathrm{NR}$ & $\mathrm{NR}$ & NR & NR \\
\hline $216^{+}$ & $\begin{array}{l}\text { New } \\
\text { born }\end{array}$ & NR & 1949 & PNG & $\begin{array}{l}\text { Western } \\
\text { Highlands }\end{array}$ & $\mathrm{NR}$ & $\mathrm{NR}$ & $\mathrm{NR}$ & $\mathrm{NR}$ & NR & NR \\
\hline $27^{\circ}$ & $\mathrm{NR}$ & $\mathrm{F}$ & 1951 & PNG & New Ireland & $\mathrm{NR}$ & Perineal \& inguinal regions & $\mathrm{NR}$ & $\mathrm{NR}$ & $\mathrm{NR}$ & $\mathrm{NR}$ \\
\hline $218^{+}$ & NR & M & 1951 & PNG & New Ireland & $\mathrm{NR}$ & Torso & $\mathrm{NR}$ & $\mathrm{NR}$ & NR & $\mathrm{NR}$ \\
\hline $219^{+}$ & 14 & M & 1961 & PNG & $\begin{array}{c}\text { Easternn } \\
\text { Highlands }\end{array}$ & $\mathrm{NR}$ & Face & NR & $\mathrm{NR}$ & NR & NR \\
\hline $220^{\dagger}$ & NR & M & 1963 & PNG & $\begin{array}{l}\text { Easternn } \\
\text { Highlands }\end{array}$ & $\mathrm{NR}$ & Ear & NR & NR & NR & NR \\
\hline $221^{+}$ & NR & F & 1963 & PNG & $\begin{array}{l}\text { Easternn } \\
\text { Highlands }\end{array}$ & $\mathrm{NR}$ & Ear & $\mathrm{NR}$ & $\mathrm{NR}$ & NR & $\mathrm{NR}$ \\
\hline $222^{+}$ & NR & $\mathrm{F}$ & 1979 & PNG & Morobe & $\mathrm{NR}$ & Eye & $\mathrm{NR}$ & $\mathrm{NR}$ & NR & $\mathrm{NR}$ \\
\hline $223^{\dagger}$ & 5 & M & 1982 & PNG & $\begin{array}{l}\text { Western } \\
\text { Province }\end{array}$ & $\mathrm{NR}$ & $\mathrm{NR}$ & $\mathrm{NR}$ & NR & NR & $\mathrm{NR}$ \\
\hline $224^{*}$ & 10 & M & 1983 & PNG & $\begin{array}{l}\text { Western } \\
\text { Province }\end{array}$ & $\mathrm{NR}$ & Scalp & $\mathrm{NR}$ & NR & $\mathrm{NR}$ & $\mathrm{NR}$ \\
\hline
\end{tabular}




\begin{tabular}{|c|c|c|c|c|c|c|c|c|c|c|c|}
\hline $225^{136}$ & NR & NR & 1982 & $\begin{array}{r}\text { Saudi } \\
\text { Arabia }\end{array}$ & NR & $\mathrm{NR}$ & Nose & NR & $\mathrm{NR}$ & NR & NR \\
\hline $226^{24}$ & 65 & M & 1985 & $\begin{array}{c}\text { Saudi } \\
\text { Arabia }\end{array}$ & NR & Low & Eye: globe & Surgical removal, antibiotics & $\begin{array}{l}\text { Multiple underlying illnesses, stroke, trauma, ulcers, bacterial infections, mental } \\
\text { illness and neurological disorder: right-sided hemiparasis and mental retardation, } \\
\text { superficial corneal pannus, stellate conjunctival scarring, and mature cataract }\end{array}$ & $\begin{array}{l}\text { Increasing pain, inflammation, discharge, swollen, noticed larvae, severe tissues } \\
\text { and/or bone destruction: totally destroyed orbit }\end{array}$ & Discharged \\
\hline $227^{137}$ & 94 & $\mathrm{~F}$ & 2014 & Thailand & Chiang Mai & Low & Eye: right orbit & Surgical removal & Bedridden, right eye infection & Right eyelid swollen, itch, ulcer, noticed larvae, bleeding, discharge, necrosis & Discharged \\
\hline $228-286^{138-139}$ & & - & 1920-1922 & India & - & - & & - & - & & - \\
\hline $287^{140}$ & - & - & 1929 & $\begin{array}{c}\text { Cote } \\
\text { d'lvoire }\end{array}$ & - & - & Face & - & - & - & - \\
\hline $288^{141}$ & - & - & 1932 & Kenya & - & - & Limb: foot & - & - & - & - \\
\hline $289^{142}$ & - & - & 1944 & Uganda & - & - & Eye: orbit & - & - & - & - \\
\hline $290^{142}$ & - & - & 1944 & Uganda & - & - & Nose & - & - & - & - \\
\hline $291^{142}$ & - & - & 1944 & Uganda & - & - & Limb: foot & - & - & - & - \\
\hline
\end{tabular}

COPD=Chronic obstructive pulmonary disease; DRC=Democratic Republic of the Congo; PNG=Pupua New Guinea; RCHE=a residential care home for the elderly

* Case recorded by Zhou et al in Guangdong Province, China

Spradbery et al Per.comm.

Seveal strikes on vavels of new born children

**reported cause of death

\section{References}

70. Center For Health Protection. A case of Chrysomya bezziana infestation in a breast mass. Communicable Disease Watch. 2005;2:2.

71. Center For Health Protection. A local case of Chrysomya bezziana infestation. Communicable Disease Watch. 2005;2:95.

72. Center For Health Protection. A case of Chrysomya bezziana infestation. Communicable Disease Watch. 2005;2:83.

73. Center for Health Protection. Public reminded to guard against Chrysomya bezziana infestation. Available from: https://www.chp.gov.hk/en/features/2178.html.

74. Center for Health Protection. A case of human Chrysomya bezziana infestation confirmed. Available from: https://www.chp.gov.hk/en/features/2506.html.

75. Center for Health Protection. A case of human myiasis under investigation. Available from: https://www.chp.gov.hk/en/features/6811.html.

76. Center for Health Protection. Case of human Chrysomya bezziana infestation under investigation. Available from: http://www.info.gov.hk/gia/general/200604/07/P200604070245.html.

77. Center For Health Protection. A case of Chrysomya bezziana infestation. Communicable Disease Watch. 2006;3:22-23.

78. Center For Health Protection. A case of human myiasis reported. Communicable Disease Watch. 2007;4:22.

79. Center For Health Protection. A confirmed case of human myiasis. Communicable Disease Watch. 2010;7:66.

80. Center For Health Protection. Two confirmed cases of human myiasis. Communicable Disease Watch. 2010;7:91.

81. Center For Health Protection. Two confirmed cases of human myiasis. Communicable Disease Watch. 2010;7:105.

82. Center For Health Protection. A confirmed case of human myiasis. Communicable Disease Watch. 2011;8:5.

83. Center For Health Protection. A case of human myiasis. Communicable Disease Watch. 2011;8:66.

84. Center For Health Protection. Two cases of human myiasis. Communicable Disease Watch. 2011;8:99.

85. Center For Health Protection. A case of human myiasis. Communicable Disease Watch. 2011;8:15.

86. Center For Health Protection. A case of human myiasis. Communicable Disease Watch. 2011;8:24.

87. Center For Health Protection. A confirmed local recurrent case of human myiasis. Communicable Disease Watch. 2012;9:1.

88. Center For Health Protection. Four sporadic cases of human myiasis. Communicable Disease Watch. 2012;9:90-91.

89. Center For Health Protection. Two cases of human myiasis. Communicable Disease Watch. 2013;10:104.

90. Center For Health Protection. A case of human myiasis. Communicable Disease Watch. 2013;10:7.

91. Center For Health Protection. A case of human myiasis.Communicable Disease Watch. 2013;10:92.

92. Center For Health Protection. A case of human myiasis. Communicable Disease Watch. 2013;10:88.

93. Center For Health Protection. A sporadic case of human myiasis. Communicable Disease Watch. 2015;12:9.

94. Center For Health Protection. A case of human myiasis.Communicable Disease Watch. 2015;12:67.

95. Center For Health Protection. A case of human myiasis.Communicable Disease Watch. 2015;12:75

96. Center For Health Protection. A confirm local case of human myiasis.Communicable Disease Watch. 2015;12:32.

97. Center For Health Protection. A cofirmed local case of human myiasi.Communicable Disease Watch. 2015;12:41

98. Center For Health Protection. A sporadic local case of human myiasis in a residential care home for the elderly (RCHE). Communicable Disease Watch. 2015;12:110

99. Center For Health Protection. Two cases of human myiasis. Communicable Disease Watch. 2016;13:7.

100. Center For Health Protection. Two local cases of human myiasis. Communicable Disease Watch. 2016;13:50.

101. Center For Health Protection. A sporadic case of human myiasis. Communicable Disease Watch. 2016;13:100

102. Center For Health Protection. A sporadic case of human myiasis. Communicable Disease Watch. 2017;14:18.

103. Center For Health Protection. Two local cases of human myiasis. Communicable Disease Watch. 2018;15(19):84.

104. Poon T. Oral myiasis in Hong Kong - a case report. HK Pract. 2006;28:388-393.

105. Chan T, Yan K, Yien L, Yuen W. Oral and cutaneous myiases caused by Chrysomya bezziana. Surgical Practice. 2010;9:28-30.

106. Lam KH, Lui TH. Myiasis of the foot and leg caused by Chrysomya bezziana. J Foot Ankle Surg. 2014;53(1):88-91. doi: 10.1053/j.jfas.2013.05.00 
107. Kwong A, Yiu WK, Chow LW, Wong S. Chrysomya bezziana: a rare infestation of the breast. Breast J. 2007;13(3):297-301. doi: 10.1111/j.1524-4741.2007.00426.x

108. Zachariah JE, Sehgal K, Dixit UB, Bhatia R. Oral myiasis: a case report. Spec Care Dentist. 2014;34(1):51-53. doi: 10.1111/scd.12016

109. Verma L, Pakrasi S, Kumar A, Sachdev MS, Mandal AK. External ophthalmomyiasis associated with herpes zoster ophthalmicus. Can J Ophthalmol. 1990;25(1):42-43.

110. Amandeep S, Devinder S, Rajiv J. Chrysomya bezziana (Diptera: Calliphoridae) as a causative agent of myiasis in patients suffering from oral cancer. Journal of Entomological Research. 2011;35:187-189.

111. Avula JK, Avula H, Arora N, Manchukonda UK, Vivekavardhan RN. Orofacial myiasis of the gingiva and nasal cavity: a report of two cases and general review. J Periodontol. 2011;82(9):1383-1388. doi: 10.1902/jop.2011.100724

112. Rathi S, Pednekar K, Pathak A, Singh P. Screw-worm myiasis of prolapsed rectum. Indian Pediatr. 2014;51(1):53-54.

113. Ghosh SK, Bandyopadhyay D, Sarkar S. Myiasis in a large perigenital seborrheic keratosis. Indian J Dermatol. 2010;55(3):305-306. doi: 10.4103/0019-5154.70699

114. Maheshwari V, Naidu S. Oral Myiasis caused by Chrysomya bezziana A case report. Peoples Journal of Scientific Research. 2010;3(2):25-26.

115. Aggarwal A, Daniel MJ, Shetty RS, Kumar BN, Sumalatha CH, Srikanth E, et al. Oral Myiasis Caused by Chrysomya bezziana in Anterior Maxilla. Case Reports in Dentistry. 2014;2014:1-4. doi: 10.1155/2014/518427

116. Jimson S, Prakash CA, Balachandran C, Raman M. Oral myiasis: case report. Indian J Dent Res. 2013;24(6):750-752. doi: 10.4103/0970-9290.127626

117. Sankari LS, Ramakrishnan K. Oral myiasis caused by Chrysomya bezziana. J Oral Maxillofac Pathol. 2010;14(1):16-18. doi: 10.4103/0973-029X.64304

118. Candamourty R, Venkatachalam S, Yuvaraj V, Sujee C. Oral myiasis in an adult associated with filariasis and Hansen's disease. J Nat Sci Biol Med. 2013;4(1):259-262. doi: 10.4103/0976-9668.107322

119. Pandhi D, Singal A, Das S. Myiasis arising in condylomata acuminata: an unusual presentation. Int J Std Aids. 2011; 22(4):238-240. https://doi.org/10.1258/jijsa.2010.010357

120. Kumar GV, Sowmya G, Shivananda S. Chrysomya bezziana oral myiasis. J Glob Infect Dis. 2011;3(4):393-395. doi: 10.4103/0974-777X.91066

121. Anand S, Taneja S. Oral myiasis, a case report. Armed Forces Med J India. 1971;27:277-278.

122. Singh A, Singh D. Traumatic Myiasis of the Scalp in a 3-year-old Patient- A Case of Neglected Health Care. Malaysian Journal of Medicine \& Health Sciences. 2019;15(1):89-91

123. Talari S, Yeganeh-Moghadam A, Dehghani R. Chrysomya bezziana infestation. Arch Iran Med. 2002;5(1):56-58.

124. Ahmadi MS, Nasirian H, Gheshmi AMN, Ershadi MRY. Human Extensive Head Skin Myiasis. Iran J Public Health. 2009;38(1):134-138

125. Jdalayer T, Maleki M, Monghtaderi M. Human urogenital myiasis caused by Chrysomyia bezziana. Iran J Public Health. 1978;7:116-117

126. El-Azazy OM. Old World screw-worm fly (Chrysomyia bezziana) in the Gulf. Vet Rec. 1993;132(10):256.

27. Alizadeh AM, Zamani N. Myiasis in an 89-year-old man with non-hodgkin lymphoma. J Arthropod Borne Dis. 2014;8(1):117-118.

128. Lee HL, Krishnasamy M, Jeffery J. A case of human nasopharyngeal myiasis caused by Chrysomya bezziana Villeneuve, 1914 (Diptera: Calliphoridae) in Malaysia. Trop Biomed. 2005;22(1):87-88.

129. Ramalingam S, Nurulhuda A, Bee LH. Urogenital myiasis caused by Chrysomya bezziana (Diptera: Calliphoridae) in peninsular Malaysia. The Southeast Asian journal of tropical medicine and public health. 1980;11(3):405-407.

130. Rohela M, Jamaiah I, Amir L, Nissapatorn V. A case of auricular myiasis in Malaysia. Southeast Asian J Trop Med Public Health. 2006;37 Suppl 3:91-94.

131. Bayer HG. Myiasis maligna of nose and ears in Ceylon; recommendation of a new treatment. AMA Arch Otolaryngol. 1954;59(1):104-107.

132. Lubis RR, Albar MY, Darlan DM. Massive orbital myiasis arising from nasal myiasis in an Indonesian patient with diabetes. American journal of ophthalmology case reports. 2019;13:147-150. doi: 10.1016j.ajoc.2019.01.006

133. Slesak G, Inthalad S, Strobel M, Marschal M, Hall MJ, Newton PN. Chromoblastomycosis after a leech bite complicated by myiasis: a case report. Bmc Infect Dis. 2011;11:14. doi: 10.1186/1471-2334-11-14

134. Zaidi F, Ali N, Khisroon M. Urinogenital Myiasis From Blow Fly in a Pakistani Child. J Coll Physicians Surg Pak. 2016;26(6 Suppl):S35-S36.

135. Fain A, Magis P, Verdin G, Donkers J, Gobbels P. [On 2 cases of human myiasis produced by Chrysomyia bezziana Villeneuve in the Belgian Congo]. Ann Soc Belg Med Trop (1920). 1959;39:763-765.

136. Ansari M, Oertley R. Nasal myiasis due to Bezzi’s blowfly (screw-worm): case report. Saudi Med J. 1982;3:275-278.

137. Ausayakhun S, Limsopatham K, Sanit S, Sukontason K, Sukontason KL. Orbital ophthalmomyiasis caused by Chrysomya bezziana in Thailand. Trop Biomed. 2018:35(1):288-292.

38. Patton W. Note on the occurrence of Passeromyia heterochaeta Villeneuve in India. Ind J Med Res. 1920;8:30.

139. Patton W. Some notes on Indian Calliphorinae VII. Additional cases of myiasis caused by the larvae of Chrysomyia bezziana Vill., together with some notes on the Diptera which cause myiasis in man and animals. Ind J Med Res. 1922;9:654.

140. Bouffard G, Legac P. Myiase a Chrysomyia bezzianum observe chez un indigene de la Cote d'Ivoire. Bull Soc Path Exot. 1929;22:48.

141. Symes C, Roberts J. A list of the Muscidae and Oestridae causing myiasis in man and animals in Kenya recorded at the medical research laboratory, Nairobi. E Afr Med J. 1932;9:18.

142. Hopkins G. Notes on myiasis especially in Uganda. E Afr Med J. 1944;21:258 